\title{
Adaptive Fault Monitoring in Fault Tolerant CORBA*
}

\author{
Soo Myoung Lee ${ }^{1}$, Hee Yong Youn ${ }^{1}$, and We Duke Cho ${ }^{2}$ \\ ${ }^{1}$ School of Information and Communications Engineering \\ SungKyunKwan University, 440-746, Suwon, Korea \\ \{lifetime, youn\}@ece.skku.ac.kr \\ ${ }^{2}$ CUCN, Suwon, Korea \\ chowdeajou.ac.kr
}

\begin{abstract}
A number of different kinds of applications developed on CORBA framework need fault tolerance in asynchronous distributed system or network environment, and it is important to quickly detect the faults. There exist various fault monitoring and detection algorithms that employ a timeout-based mechanism. However, they are occasionally inaccurate in unstable or overloaded system. The goal of the proposed algorithm is to enhance the accuracy of fault monitoring. This is achieved by promptly adjusting the timeout interval using the past elapsed time values accumulated. Additionally, we use asynchronous invocation to call 'is_alive()' method of monitorable object with a sequence number. Experiment on CORBA-compliant Orbix ORB confirms the effectiveness of the proposed scheme compared to the existing one.
\end{abstract}

Keywords: Adaptation, elapsed time, fault monitoring and detection, faulttolerant CORBA, timeout.

\section{Introduction}

A number of different kinds of applications developed by the members of the OMG and users of CORBA [1] need fault tolerance features. Fault-tolerant CORBA [2] aims to provide robust support for the applications requiring high reliability. Here it is very important to quickly detect a fault if it occurs. We call the mechanism detecting any fault occurrence as fault monitoring and detection. The mechanism is primarily based on timeout event occurring when a request message sent from a monitor to the monitorable object does not return in time.

In asynchronous distributed systems it is hard to differentiate between real crash and network overload. The design of fault detector must be careful because a timeout value much smaller than the average transmission time will cause false alarm, while a timeout value much greater than the average transmission time will delay the fault detection when a crash occurs.

There are various fault detection models such as Crash-Recovery Model [3] and the model of Chandra and Toueg [4]. They suggest a way that increases the timeout

* This research was supported by the Ubiquitous Autonomic Computing and Network Project, $21^{\text {st }}$ Century Frontier R\&D Program in Korea and the Brain Korea 21 Project in 2004. Corresponding author: Hee Yong Youn 
value when a false fault or crash alarm occurred. However, this strategy allows a scenario where the timeout value is increased several times due to overload in the network. It can lead to an unnecessarily long timeout value even after the network returns to normal workload condition. The ADAPTATION-Algorithm proposed by Sotoma and Mauro Madeira [5] presents a fault detection mechanism that aims at accurate failure detection in spite of network overloads by means of periodic increases and decreases of the timeout value. However, it may deduce incorrect timeout values and monitoring intervals in unstable system or network environment because it regulates timeout values only after timeout or response event occurs and initializes several factors used for calculating the mean values.

We thus propose a solution that can solve the problem above. First of all, it employs an asynchronous invocation approach in which 'is_alive()' message is transmitted with a sequence number to the monitorable object and buffer is used that can reserve a number of measured time values for calculating several factors. Additionally, it uses some equations in the algorithm for achieving better result. Experiment on an actual client and server system reveals that the proposed algorithm allows more accurate prediction of monitoring interval than the ADAPTATION algorithm regardless of load condition of the system.

The rest of the paper is organized as follows. Section 2 presents an overview of Fault-tolerant CORBA and related work. Section 3 describes the proposed scheme. Section 4 evaluates the performance of the proposed scheme and compares it with the ADAPTATION algorithm. Finally, Section 5 concludes the paper.

\section{Background}

In fault-tolerant CORBA, fault management encompasses the following activities; fault detection: detecting the presence of a fault in the system and generating a fault report, fault notification: propagating fault reports to the entities registered for such notifications, fault analysis/diagnosis: analyzing a (potentially large) number of related fault reports and generating condensed or summary reports.

\subsection{The Models for Fault Monitoring}

Most implementations of fault detectors are based on timeout, and use either pull or push-based monitoring. Because push-based monitoring depends on the characteristics of the application, it is not defined in Fault-tolerant CORBA specification.

- Pull model: The fault monitor periodically calls 'is_alive()' method of the monitorable object asking whether it is alive. If the monitorable object does not reply within some time interval, then the fault monitor suspects it is faulty. The main advantage is that it allows a status check only when it is needed by the application.

- Push model: The monitorable object periodically calls 'i_am_alive()' method of the fault monitor informing that it is still alive. If the monitorable object has not called the 'i_am_alive()' method within some time interval, then the fault monitor suspects it is faulty. The main advantage is fast detection of the failure of the monitorable object. 


\subsection{Related Works}

The design and verification of fault-tolerant distributed applications are generally viewed as complex endeavor. In recent years, several paradigms have been identified which simplify the task. The model using the weakest failure detector [6] determines what information about failures is necessary and sufficient to solve the Consensus problem in asynchronous distributed systems subject to crash failures. In the model any failure detector has to provide at least as much information as $\diamond \mathrm{W}$. Thus, $\diamond \mathrm{W}$ is indeed the weakest failure detector for solving the Consensus problem in asynchronous systems with a majority of correct processes.

The hybrid approach [7] presents a consensus algorithm that combines randomization and unreliable failure detection, two well-known techniques solving the Consensus problem. This hybrid algorithm combines the advantages of both the approaches; it guarantees deterministic termination if the failure detector is accurate and probabilistic termination otherwise.

The model using the heartbeat failure detector [8] considers partitionable networks with process crashes and lossy links, and focuses on the problems of reliable communication and consensus for such networks. The model solves the problem by using $\diamond \mathrm{S}$ and the quiescent, i.e., algorithms that eventually stop sending messages.

In addition to the fault monitoring mechanisms, a framework called DOORS [9] was proposed, which was developed prior to the FT-CORBA standard as an experimental Fault-tolerant CORBA middleware. It was implemented as a CORBA service to provide end-to-end application-level fault tolerance.

\subsection{Detection Problem}

To accurately design a fault detection mechanism, we should avoid some situations. Figure 1 shows two examples of inaccurate timeout.

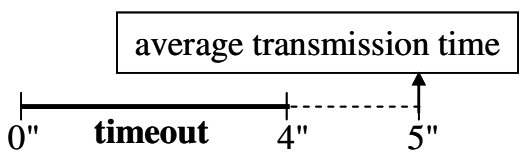

(a) Incorrect timeout event

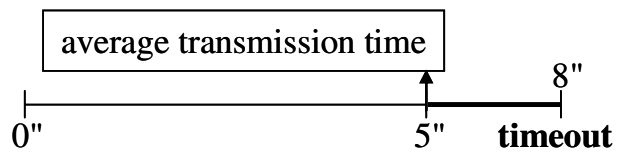

(b) Delayed timeout event

Fig. 1. Examples of inaccurate fault monitoring

As shown in Figure 1(a), if timeout occurs in 4 seconds when the average transmission time from a monitor to the monitorable object is 5 seconds, the system assumes a fault. On the other hand, as shown in Figure 1(b), if timeout occurs in 8 seconds, the system has 3 second latency in detecting a fault and this may cause a lot of fatal problems. Thus, Chandra and Toueg suggest an increase on the timeout value after a false crash alarm. However, this strategy may cause the timeout value to be increased several times due to overload in the network. It can lead to an unnecessarily long timeout value too long even after the network returns to normal workload condition. 


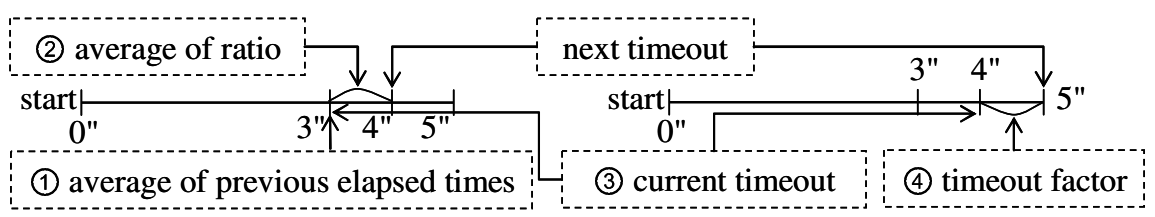

(a) RESPONSE event

(b) TIMEOUT event

Fig. 2. The operational mechanism of the ADAPTATION algorithm

Figure 2 shows the solution of the ADAPTATION algorithm toward the problem mentioned above. It regulates the timeout value using the RESPONSE and TIMEOUT event. If the RESPONSE event occurs, the algorithm decreases the next timeout value by the product of (1) and (2) (the average of the ratios of the two successive elapsed time pairs). On the other hand, with TIMEOUT event, the algorithm sets the next timeout value by the product of (3) and (4) (the timeout factor set by the user). This algorithm also shows slow reaction time and possibly incorrect timeout value because it regulates timeout value only after a timeout event occurs or a few response events occur. We next present the proposed scheme solving these problems.

\section{The Proposed Scheme}

The proposed scheme employs some new features to solve the problems mentioned above. First of all, it uses asynchronous invocation to transmit 'is_alive()' message with a sequence number to the monitorable object. Figure 3 presents how to judge normal or abnormal state such as loss or delay of the request message using the sequence number. Here successful and failed transmission/reception of a message are marked as black and white dot, respectively.

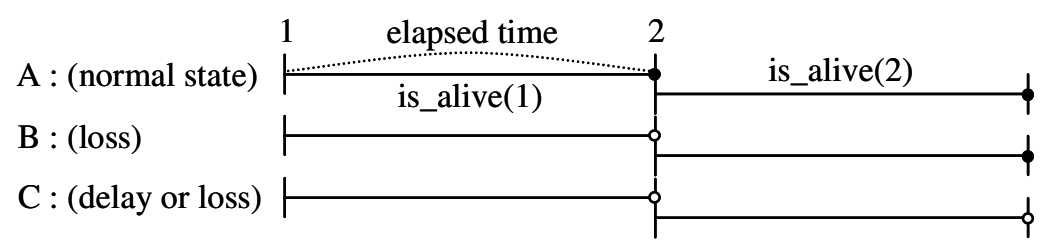

Fig. 3. Judging the state of a monitorable object

Here Case A is normal state because 'is_alive(2)' is transmitted after 'is_alive(1)' arrives. However, Case B presents loss of 'is_alive(1)' because 'is_alive(2)' arrives before 'is_alive(1)' arrives. Hence there is a need to regulate the monitoring interval and notify the state to the Notifier. At last, nobody can judge the state of the monitorable object for Case C because both 'is_alive(1)' and 'is_alive(2)' have not arrived. This is due to delay or loss.

Another feature of the proposed scheme is to promptly adjust the timeout value using the past elapsed times. For example, assume that there is a system with capri- 
ciously varying workload. To distinguish failed object from overly workload when an abnormal state is identified, the proposed monitor keeps a number of sequence numbers, round-trip times (RTT), etc. in its buffer. Here the size of a buffer is 140bytes and there exist 1000 of them. The asynchronous invocation and buffer allow the proposed scheme to outperform the existing schemes including the ADAPTATION algorithm.

The following variables are used in the proposed algorithm. seqNum: the sequence number attached to each packet; monInter: predicted monitoring interval time; sTime: the time the message is sent; rTime: the time the response is received; eTime: the elapsed time obtained by subtracting sTime from rTime; message: the character type pointer variable with "SET" or "CHANGE" value; ratio: the ratio of the current elapsed time to the previous elapsed time; pCount: the number of previous contiguously increasing or decreasing ratio values; weight: the weight of a ratio; avg_ratio: the means of ratio values. The followings are the monitor and monitorable object process.

process monitor object:

01 monInter[0] = the initial monitoring interval;

02 while(do monitoring)\{

03 check if all previous packets have returned;

04 seqNum $=$ seqNum +1 ;

05 sTime[seqNum] = timer;

06 if(call asynchronously an 'is_alive(seqNum)')\{

07 rTime [seqNum] = timer;

08 eTime [seqNum] = rTime $[$ seqNum $]-\operatorname{sTime}[$ seqNum $]$;

09 MonitoringInterval(seqNum, "SET or CHANGE");

10 \}else \{

11 send a notification to Notifier; $\}$

$12\}\}$

process monitorable object:

is_alive():

01 if(received request message from monitor)

02 return true;

The monitor process measures the initial round trip time and saves it at monInter[0] in the $1^{\text {st }}$ line. The $3^{\text {rd }}$ line is for checking the state of the monitorable object if it is in state $\mathrm{A}, \mathrm{B}$, or $\mathrm{C}$ of Figure 3 . In the $6^{\text {th }}$ line, the monitor calls 'is_alive()' method asynchronously. If any response does not return, it sends a notification to its notifier. The MonitoringInterval() function at the $9^{\text {th }}$ line regulates the monitoring interval. Here two messages are used, one for calculating the next packet's monitoring interval and another for recalculating it according to the status of returning message. The MonitoringInterval() function is as follows.

process MonitoringInterval(int seqNum, char* message):

01 if $($ message $==$ "SET" $)\{$

02 set next monitoring interval;

03 \}else if (message $==$ "CHANGE") \{

04 if(increase or decrease continually more twice) \{ 


$$
\begin{aligned}
& \text { if }(\mathrm{pCount} / 2==0) \text { weight }[\mathrm{n}-1]=(1) \text {; } \\
& \text { else weight }[\mathrm{n}-1]=(2) \text {; } \\
& \text { total }=1 \text {; } \\
& 08 \text { for(int } \mathrm{i}=\mathrm{n}-2 ; \mathrm{i}>=0 ; \mathrm{i}--)\{ \\
& 09 \text { total }=\text { total }- \text { weight }[i+1] \text {; } \\
& 10 \text { if }(\mathrm{i} !=0) \text { weight }[\mathrm{i}]=\text { total } * \text { weight }[\mathrm{i}-1] \text {; } \\
& 11 \text { else weight }[\mathrm{i}]=\text { total; }\} \text { \} } \\
& 12 \text { monInter[seqNum] = eTime[seqNum-1 }]^{*}(3) \\
& 13 \text { \}else if(after once increased, once decreased or vice versa) \{ } \\
& 14 \text { monInter[seqNum] = Regulating equation(4); } \\
& 15 \text { ratio[seqNum] = eTime[seqNum] }- \text { eTime[seqNum-1];\} }
\end{aligned}
$$

The MonitoringInterval process has two parameters. The sequence number identifies the packet called the 'is_alive()' method, and the message parameter of a character type pointer variable indicates the condition whether it establishes next monitoring interval or updates current monitoring interval. The message that has a "SET" value sets next monitoring interval, while "CHANGE" value asynchronously reflects the result calculated by the following equations to the current monitoring interval. This process is to distinguish the case that the elapsed time increases or decreases continuously from the case that the elapsed time increases and then decreases next or vice versa.

$$
\begin{gathered}
\frac{n+4}{4 n},(n=2 n) \\
\frac{n+1}{2 n},(n=2 n-1) \\
\text { avg_ratio }=\frac{\sum_{i=0}^{p C \text { count }-1} \text { eTime } e_{i} \times \text { weight }_{i}}{p \text { Count }},(\text { The pCount is an integer larger than 1.) } \\
\text { Regulating }=\text { eTime }_{0}+\frac{\sum_{i=0}^{1} \text { eTime }}{2}
\end{gathered}
$$

Equation (1) and (2) are used when the last elapsed time increases and decreases continuously more than twice, respectively. Equation (3) is used to get the average ratio. Equation (4) is to regulate the monitoring interval in the case that the elapsed time increases and then decreases next or vice versa.

\section{Performance Evaluation}

In this section the proposed scheme is evaluated by experiment and compared with the ADAPTATION algorithm to display what factor allows better monitoring interval according to the system workload changed per second. The client and server system used for the experiment have Intel Pentium 4 processor and 1GB main memory. The OS is Windows XP with established CORBA-compliant Orbix Enterprise Version 6.1 $[11,12]$ and Visual $\mathrm{C}++$ 6.0. The monitor and monitorable object program reside in 
different systems. We use two sets of delay values $\{0,0.01,0.1,1\}$ and $\{0,0.01,0.1$, $1,1.5,2.5,5,10\}$ that represent the response delay time for the request message caused by changing the system workload for comparing the performances of the algorithms in stable and unstable condition, respectively. We assume that initial monitoring interval is 1.2 microsecond. Also, in order to cause changing elapsed time, the workload is gradually increased in the first 4 second period and then decreased in the next 4 second period repeatedly.

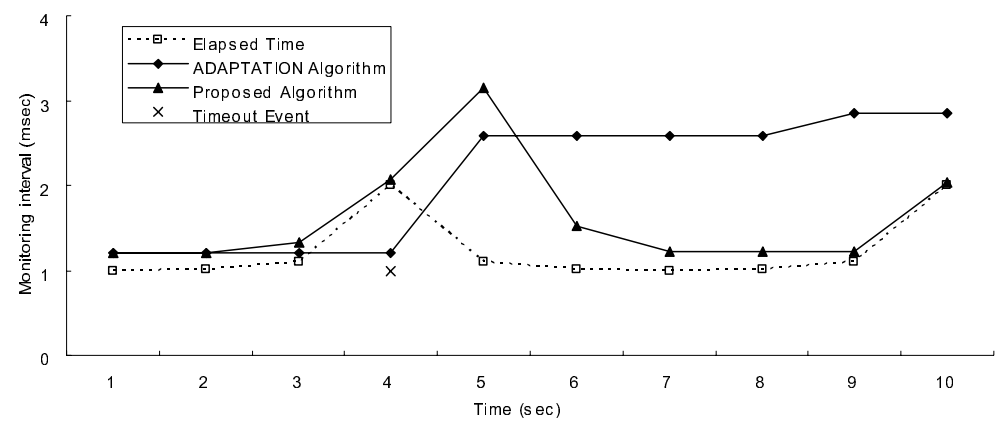

Fig. 4. The monitoring intervals as time moves in stable condition

Figure 4 displays the changing monitoring intervals of the two algorithms as the system workload changes in stable system. Notice that the line of elapsed time (the dotted line) does not vary a lot in stable condition, while the monitoring interval regulated by the proposed algorithm (the line with triangular dots) chases the elapsed time trajectory quite fast. However, the monitoring interval regulated by the ADAPTATION algorithm(the line with lozenge dots) is not as prompt as the proposed algorithm. Particularly, it has incorrect monitoring interval when time is 4 (in other words the interval is shorter than the elapsed time). In spite the monitoring interval should decrease if the elapsed time decreases, the monitoring interval regulated by the ADAPTATION algorithm shows little change in contrast with the proposed algorithm.

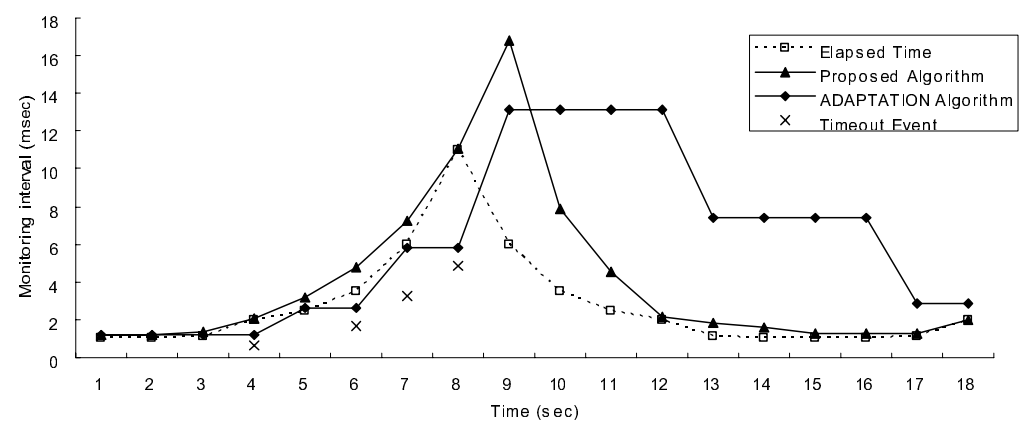

Fig. 5. The monitoring intervals as time moves in unstable condition 
Figure 5 presents the changing monitoring intervals of the two algorithms as the system workload changes in unstable system or network environment. The experiment results of the two algorithms are similar to Figure 4. Notice that, in spite of the system workload increasing rapidly, the proposed algorithm chases the trajectory of the elapsed time fast. The ADAPTATION algorithm is worse in the unstable condition.

\section{Conclusion}

A number of different kinds of applications developed on CORBA framework in asynchronous distributed system or network environment need fault tolerance. Particularly, in real-time distributed processing systems, it is important to quickly detect the fault and properly adjust the monitoring interval in which the 'is_alive()' message is predicted to return. This paper has presented a new fault monitoring algorithm that uses elapsed time values to decide the monitoring interval. Also, it has an important feature of asynchronous invocation with sequence number.

Experiment was performed on a client and server system presenting varied monitoring interval according to the changed system workload in stable and unstable condition, respectively. The proposed algorithm allows monitoring interval close to the elapsed times while the ADAPTATION algorithm does not. Also, few timeout events occurred with the proposed algorithm in unstable system or network situation unlike the ADAPTATION algorithm. The monitoring interval must be larger than the elapsed time and the proposed algorithm allows it. A more fine tuning of the proposed approach will be carried out in the future.

\section{References}

1. Object Management Group, The Common Object Request Broker Architecture: Core Specification Version 3.0.3-Editorial changes formal/04-03-12, 2004

2. Object Management Group, Fault Tolerant CORBA, OMG document: formal/04-03-12, 2004

3. M. K. Aguilera, W. Chen and S. Toueg. Failure Detection and Consensus in the CrashRecovery Model. Technical Report 98-1676, Department of Computer Science, Cornell University, 1998.

4. T. D. Chandra and S. Toueg. Unreliable failure detectors for reliable distributed systems. Journal of the ACM, 43(2):225-267, 1996

5. Sotoma, I. and Mauro Madeira, E.R. ADAPTATION-Algorithms to ADAPTive FaulT MonItOriNg and Their Implementation on CORBA. Distributed Objects and Applications, 2001.

6. T. D. Chandra, V. Hadzilacos and S. Toueg. The Weakest Failure Detector for Solving Consensus. Journal of the ACM, 43(4):685-722, 1996

7. M. K. Aguilera and S. Toueg, Randomization and Failure Detection: A Hybrid Approach to Solve Consensus. SIAM Journal on Computing, 28(3):890-903, 1999

8. M. K. Aguilera, W. Chen and S. Toueg. Using the Heartbeat Failure Detector for Quiescent Reliable Communication and Consensus in Partitionable Networks, Theoretical Computer Science, Volume 220(1): 3-30, 1999 
9. B. Natarajan, A. Gokhale and D. C. Schmidt, DOORS: Towards High-performance FaultTolerant CORBA, Proceedings of the $2^{\text {nd }}$ International Symposium on Distributed Objects and Applications (DOA2000), 2000

10. D. Szentivanyi and S. Nadjm-Tehrani, Building and Evaluating a Fault-Tolerant CORBA Infrastructure, in Proceedings of the Workshop on Dependable Middleware-Based Systems (WDMS'02) - part of the International Conference on Dependable Systems and Networks $(D S N), 2002$

11. IONA, Orbix ORB Enterprise Version 6.1 with Visual C++ Development Toolkits, http://www.iona.com/downloads/, 2004

12. IONA, "Orbix CORBA Programmer's Guide C++ Edition, Version 6.1”, 2003 